\section{ORIGINAL RESEARCH}

R.I. Farb

R. Forghani

S.K. Lee

D.J. Mikulis

R. Agid

\title{
The Venous Distension Sign: A Diagnostic Sign of Intracranial Hypotension at MR Imaging of the Brain
}

\begin{abstract}
BACKGROUND AND PURPOSE: Patients with intracranial hypotension $(\mathrm{IH})$ demonstrate intracranial venous enlargement with a characteristic change in contour of the transverse sinus seen on routine T1-weighted sagittal imaging. In $\mathrm{IH}$, the inferior margin of the midportion of the dominant transverse sinus acquires a distended convex appearance; we have termed this the venous distension sign (VDS). This is distinct from the normal appearance of this segment, which usually has a slightly concave or straight lower margin. This sign is introduced, and its performance as a test for the presence of this disease is evaluated.
\end{abstract}

MATERIALS AND METHODS: The transverse sinuses on T1-weighted sagittal imaging of 15 patients with $\mathrm{IH}$ and 15 control patients were independently assessed in a blinded fashion by 3 readers for the presence of a VDS. A present or absent VDS was determined for each patient by each reader, and a consensus result for each patient was determined by unanimity or majority rule.

RESULTS: Using the VDS, the readers correctly identified 93\% (14 of 15) of the IH patients and similarly $93 \%$ (14 of 15) of the control patients. There was a high rate of agreement among the readers for the interpretation of the VDS (multirater $\kappa=0.82$ ). The overall sensitivity of the VDS for the diagnosis of intracranial hypotension was $94 \%$. Specificity was also $94 \%$.

CONCLUSION: The VDS appears to be an accurate test for the presence or absence of $\mathrm{IH}$ and may be helpful in the evaluation of these patients.

ntracranial hypotension ( $\mathrm{IH})$ is a syndrome of variable etiologies, clinical presentations, and MR imaging appearances that share a unifying cause of CSF volume depletion and resultant low CSF hydrostatic pressure. ${ }^{1-7}$ The syndrome of spontaneous $\mathrm{IH}(\mathrm{SIH})$ typically presents with orthostatic headache associated with MR findings and most commonly results from spontaneous leak of CSF from the spinal thecal sac. Iatrogenic causes of IH include "over shunting" associated with CSF diversion procedures, as well as continued leaks after surgical procedures and lumbar punctures. Many characteristic MR findings have been associated with $\mathrm{IH}$ regardless of its etiology. $^{1-12}$

On review of the MR imaging of patients presenting to our institution with $\mathrm{IH}$, we have identified a highly reliable novel imaging sign for the diagnosis of $\mathrm{IH}$. We have termed this sign the "venous distension sign" (VDS) and evaluated for it on routine nonenhanced sagittal T1-weighted imaging of the brain. In patients with $\mathrm{IH}$, the dural sinuses enlarge as they compensate for

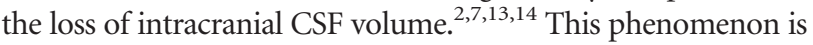
not easily quantified; however, the expansion results in a characteristic change in the contour of the dural sinuses. This contour change is readily appreciated at the level of the transverse sinus on standard nonenhanced T1-weighted sagittal images of the brain. The presence of this sign can be determined in a binary fashion. Specifically, when visualized in its midportion on sagittal images of the brain, the contour of the dominant (larger) transverse sinus

Received December 5, 2006; accepted after revision January 29, 2007.

From the Department of Medical Imaging (R.I.F., S.K.L., D.J.M., R.A.), Division of Neuroradiology, Toronto Western Hospital, University of Toronto, Toronto, Ontario, Canada; and Department of Medical Imaging (R.F.), McGill University, Montreal, Quebec, Canada.

This research was supported by the Department of Medical Imaging, University of Toronto.

Please address correspondence to Richard I. Farb, Division of Neuroradiology, Department of Medical Imaging, University Health Network, Toronto Western Hospital, New East Wing 3MC 430, 399 Bathurst St, Toronto, Ontario, M5T 2S8, Canada; e-mail: richard.farb@utoronto.ca

DOI 10.3174/ajnr.A0621 normally has a concave or straight inferior border (Fig 1). In cases of $\mathrm{IH}$, the inferior border acquires a distended appearance with a convex bulging of its inferior border (a VDS, as in Figs 2 and 3). The purpose of this paper is to report this sign and evaluate its performance in a controlled trial for identifying patients with $\mathrm{IH}$ and differentiating them from normal age- and sex-matched control subjects.

\section{Materials and Methods}

Institutional review board approval was obtained for this project.

\section{Patients with IH}

A retrospective review of clinical and imaging records was carried out to identify patients evaluated for IH at our institution from February 2001 to September 2005. Criteria used in this study to firmly establish the diagnosis of IH were similar to those reported previously for $\mathrm{SIH}^{2,15}$ and include the following: diffuse headache that worsens within 15 minutes after sitting or standing; evidence of low CSF pressure on MR imaging; and spontaneous headache resolution or within several days after epidural blood patch (EBP). Fifteen IH patients (IHPs) with complete MR imaging at presentation were identified and included 13 women and 2 men with an overall average age of 46 years. Of the 15 patients with confirmed IH, 12 presented with primary SIH, 2 presented after lumbar puncture, and 1 after a spinal anesthetic procedure. All 15 of the IHPs provided the typical clinical presentation of orthostatic headaches, as well as characteristic findings at gadolinium-enhanced MR imaging of the brain. Nine of the 15 went on to have EBP treatment, 4 had spontaneous resolution of their symptoms, and 2 were lost to follow-up. Follow-up MR imaging of the brain was available in 10 of the 15 IHPs.

\section{Control Patients}

Eighty consecutive potential control patients (CPs) were recruited from the population of cancer patients at our institution who were undergoing screening for intracranial metastatic disease. These patients had no clinical signs or symptoms of neurologic disease. 

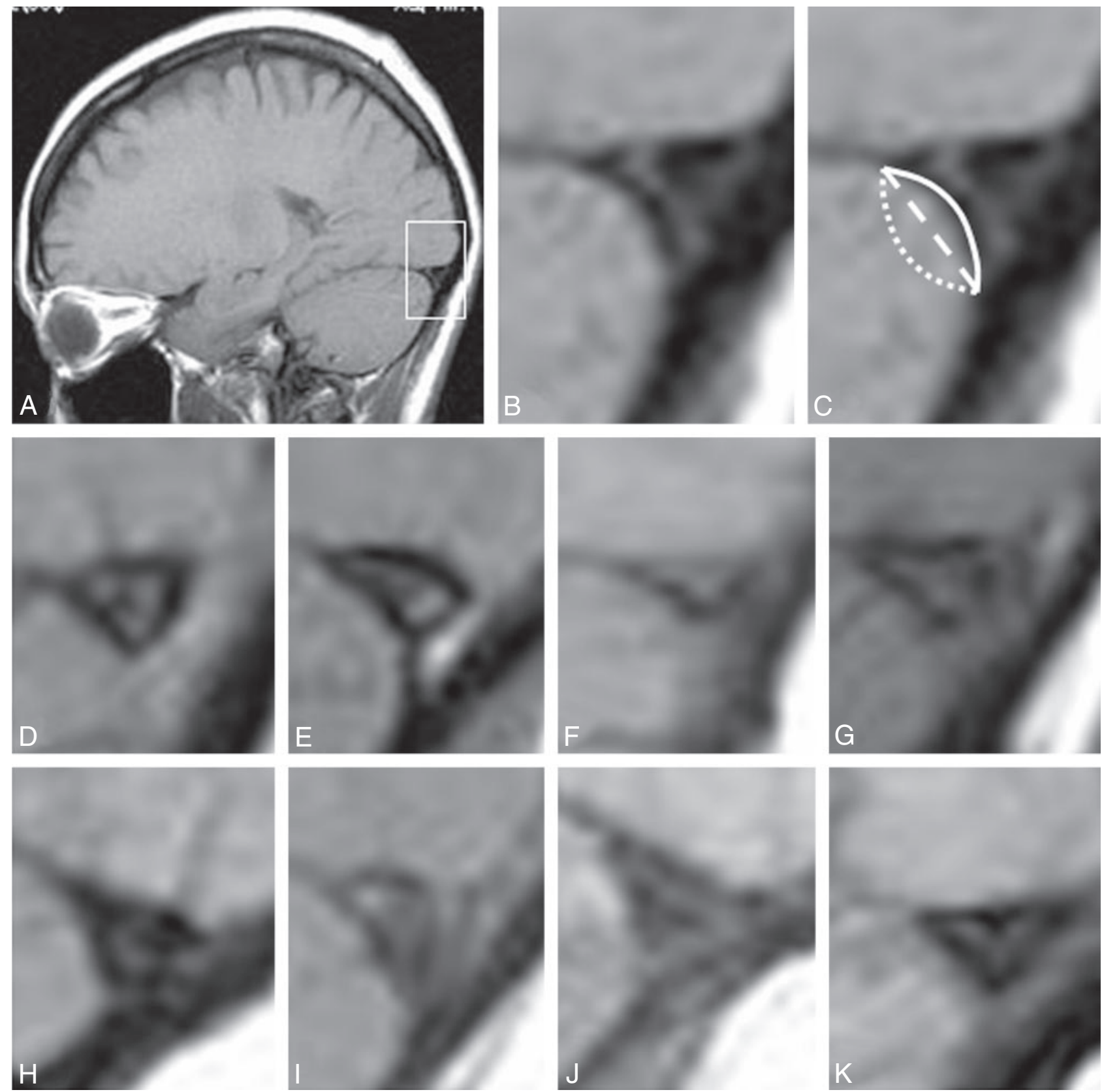

Fig 1. Normal contour of the transverse sinus. $A$, T1-weighted image through the approximated middle third of the dominant transverse sinus in a normal control. $B$, Magnified and cropped image of boxed area in A. C, Same image as $B$, with schematic representations of the inferior border of the transverse sinus drawn on the image. Both solid and dashed lines represent the normal variability in the contour of this lower border as demonstrated in individual normal controls $D$ through $K$. The dotted line in $C$ depicts the distended, convex, lower border of the middle third of the dominant transverse sinus (the VDS) as seen in intracranial hypotension (Fig 3).

At gadolinium-enhanced MR imaging, 21 of these patients had intracranial abnormalities (metastatic disease) and were excluded from the study. Images from 3 of the CPs were no longer available for review in this study. The preliminary control population consisted of 56 patients with an average age of 58 years and a femaleto-male ratio of 1:0.9. A final control population of 15 age-approximated control subjects was derived from the larger control population by sequestering the youngest patients available within the preliminary control population. This control population included 13 women and 2 men, with an overall average age of 51 years. Informed consent was obtained from all of the control subjects before their participation.

\section{MR Imaging, Processing, and Review}

All of the MR examinations were carried out with a superconducting 1.5T MR system (Signa EchoSpeed 8.2.3-11.2 software; GE Medical Systems, Milwaukee, Wis) with a standard head coil. All of the examinations included a T1-weighted sagittal conventional spin-echo (CSE) sequence (TR/TE, $600 \mathrm{~ms} / 14 \mathrm{~ms}$; section thickness, $5 \mathrm{~mm}$ with an intersection gap of $2 \mathrm{~mm}$; and frequency- and phase-encoding matrix, $256 \times 192$ ). This T1-weighted sagittal CSE sequence was the only sequence used for this study. Images of this sequence for each $\mathrm{IH}$ and CP were transferred to a PACS viewing workstation (Merge efilm 1.9.4, Milwaukee, Wis). Three neuroradiologists (R.A., S.K.L., D.J.M.) acted as readers for this study. Each reader was independently 

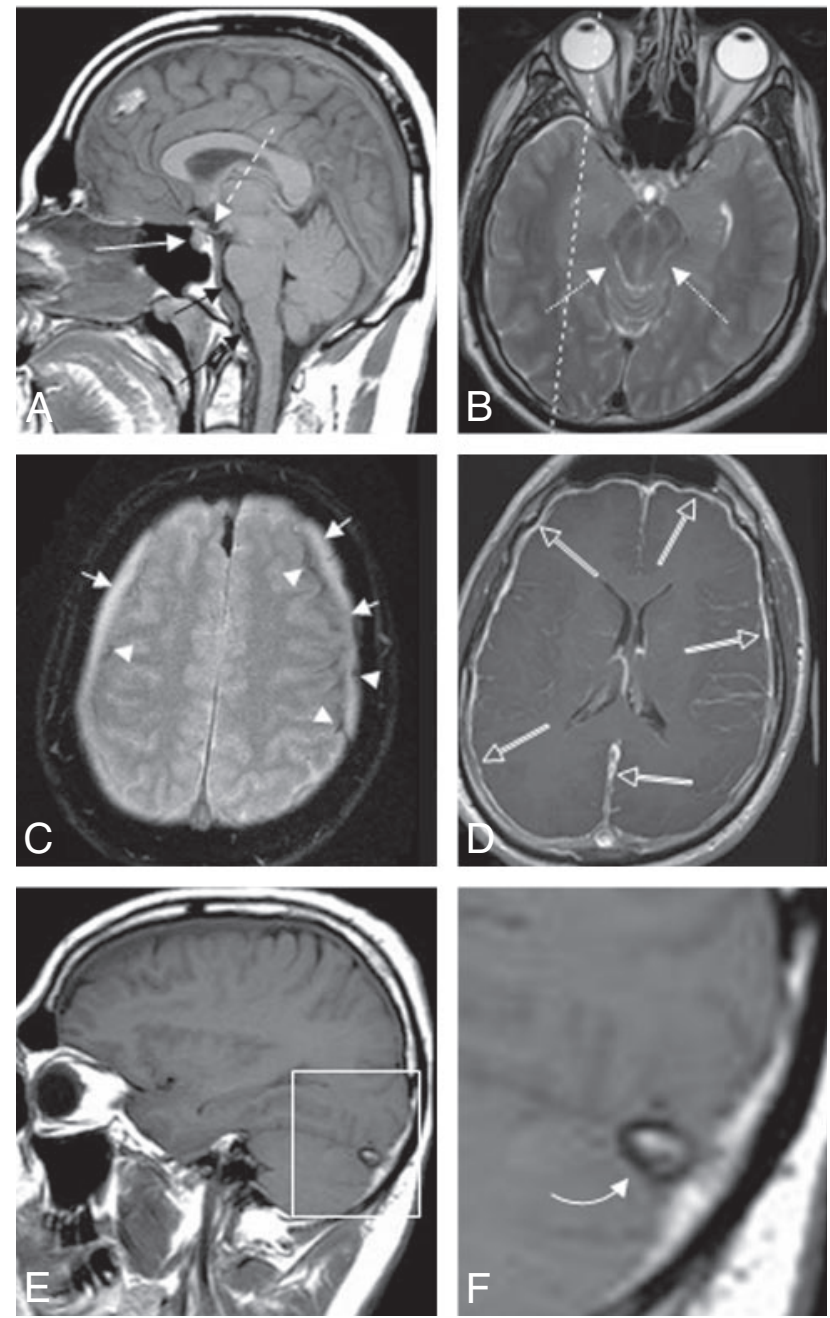

Fig 2. Typical MR findings in $I H$. A, Midline sagittal T1-weighted image showing the "sagging brain" appearance with distortion of the anterior margin of the pons and medulla (black arrows) and decreased vertical dimension of the suprasellar cistern and sagging tuber cinereum (dashed arrow), as well as the prominent pituitary gland (white arrow). B, Axial T2-weighted image showing effacement of the perimesencephalic cistern (dotted arrows), as well as the elongated anteroposterior dimension of the midbrain. $C$, Axial T2-weighted gradient echo (susceptibility weighted) image showing small bilateral subdural effusions (short arrows) with a small amount of hemosiderin staining (arrowheads), indicating previous subdural hemorrhage. D, Gadolinium-enhanced axial T1-weighted image demonstrating diffuse pachymeningeal (dural) enhancement (open arrows). E, T1weighted image through the approximated middle third of the dominant transverse sinus (located by dashed line in $B$ ). $F$, Magnified image of boxed area in $E$ showing the convex inferior margin of the transverse sinus (curved arrow), that is, the VDS indicative of $\mathrm{IH}$.

trained for several minutes on the use of the VDS by the principal author (R.I.F.) and study coordinator (R.F.) by using examples of control and confirmed IHPs not otherwise used in any portion of the study. To standardize the application and interpretation of this sign, the larger (dominant) of the 2 transverse sinuses was used. The middle third of this dominant sinus was approximated to ensure that contour evaluations were not influenced by proximity of the torcula herophili or the sigmoid sinus. The reader was asked to tabulate whether the VDS was present or absent for each image set. The readers were told that they would be evaluating a total of 30 case subjects but were given no indication regarding the number of IHPs versus normal CPs in the randomly assigned grouping. Each of the 15 CPs and 15 IHPs were assigned an identifying number and were then mixed with a digital randomizer. Images were displayed on the viewing workstation with the image cropped such that the readers could easily scroll through
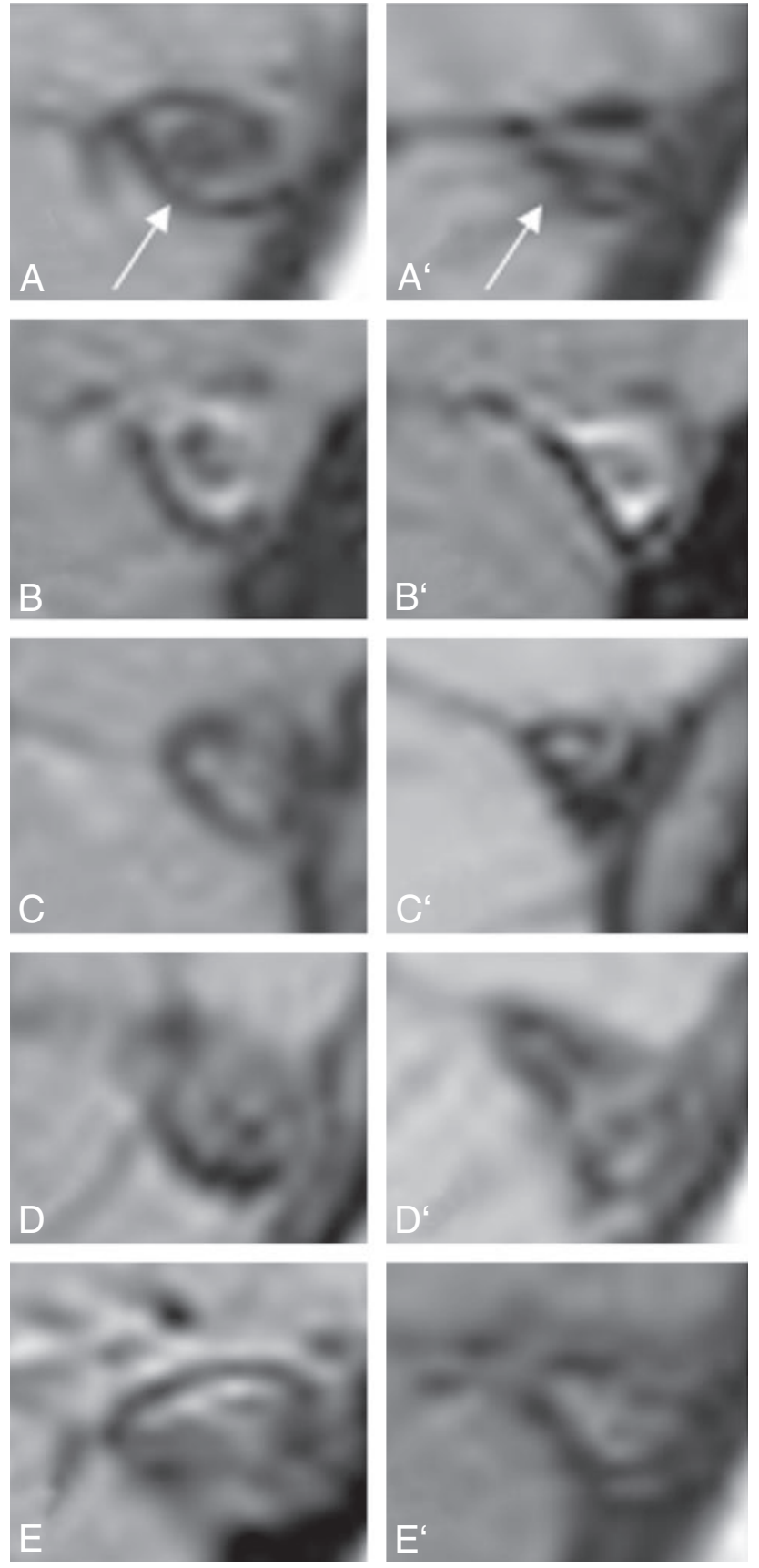

Fig 3. Examples of the VDS in 5 patients with $\mathrm{HH}$ pretreatment and posttreatment with EBP. $A-E$, Magnified and cropped sagittal T1-weighted images through the approximated middle third of the dominant transverse sinus of IHPs $1,5,11,13$, and 14 at presentation. $A^{\prime}-E^{\prime}$, Corresponding image of each patient at follow-up MR imaging, after EBP and resolution of symptoms. Note the change in the contour of the transverse sinus (particularly the inferior border) between the before and after images of each patient (arrows).

the sequential images of each patient's T1 sagittal sequence. In this manner, only the right and left transverse sinuses and little else of the intracranial anatomy were visible to the readers, thus minimizing the possibility that the readers could inadvertently identify other imaging cues of IH. All of the patient information was removed from the images. The review sessions were prepared in this masked (blinded) manner by the study coordinator (R.F.). At each review session, the reader was assisted by the study coordinator to ensure that patient identifiers were removed from the images, the data forms were completed, and the protocol of image presentation was maintained. Care 


\begin{tabular}{llllll}
\hline \multicolumn{7}{l}{ Table 1: Patients with intracranial hypotension } \\
\hline Patient & Etiology & EBP & R1 & R2 & R3 \\
\hline HP 1 & SIH & Y & pos & pos & pos \\
HP 2 & SIH & N & pos & pos & pos \\
HP 3 & SP AN & N & pos & pos & pos \\
HP 4 & SIH & Y & neg & pos & pos \\
HP 5 & Post-LP & Y & pos & pos & pos \\
HP 6 & SIH & N & pos & pos & pos \\
HP 7 & SIH & Y & pos & pos & pos \\
HP 8 & SIH & Y & pos & pos & pos \\
HP 9 & SIH & N & pos & pos & pos \\
HP 10 & SIH & N & pos & neg & neg \\
HP 11 & SIH & Y & pos & pos & pos \\
HP 12 & SIH & N & pos & neg & pos \\
HP 13 & SIH & Y & pos & pos & pos \\
HP 14 & SIH & Y & pos & pos & pos \\
HP 15 & Post-LP & Y & pos & pos & pos \\
\hline
\end{tabular}

Note:-SIH indicates spontaneous intracranial hypotension; pos, positive; neg, negative; $Y$ yes; $N$, no: $S P$ AN spinal anesthetic procedure: EBP, epidural blood patch was performed; R1, R2, R3, the interpretation of the venous distension sign by each reader for each patient; HP hypotension patient; Post-LP, after lumbar puncture.

\begin{tabular}{llll}
\hline \multicolumn{2}{l}{ Table 2: Control patients } & & \\
\hline Control & R1 & R2 & R3 \\
Subject & pos & pos & pos \\
\hline CP 1 & neg & neg & neg \\
CP 2 & neg & neg & neg \\
CP 3 & neg & neg & neg \\
CP 4 & neg & pos & neg \\
CP 5 & neg & neg & neg \\
CP 6 & neg & neg & neg \\
CP 7 & neg & neg & neg \\
CP 8 & neg & neg & neg \\
CP 9 & neg & neg & neg \\
CP 10 & neg & neg & neg \\
CP 11 11 & neg & neg \\
CP 12 & neg & neg & neg \\
CP 13 & neg & neg & neg \\
CP 14 & neg & neg & neg \\
CP 15 & neg & neg & . \\
\hline
\end{tabular}

Note:-CP indicates control patient; pos, positive; neg, negative; R1, R2, R3, the interpretation of the venous distension sign by each reader for each patient.

was taken to ensure that R.F. did not inadvertently impart information that would assist or influence the readers in their interpretations. Each reader independently reviewed the 30 sets of images. The results of the 3 readers were grouped for each patient, and a consensus response was derived based on the majority opinion ( 2 of 3 ) for response to the binary type question.

\section{Results}

The results of the study are tabulated in Tables 1 and 2. The normal contour of the middle segment of the transverse sinus on sagittal imaging was surprisingly consistent, displaying a variable contour of the upper border, a slightly convex posterior (calvarial) border, and a concave or straight inferior border as shown in Fig 1 (an absent VDS). This contour of the midtransverse sinus was seen in 14 of the 15 CPs. Conversely, in 14 of the 15 patients with $\mathrm{IH}$, the inferior border of the midtransverse sinus displayed a convex margin (a VDS). In all 10 of the patients with MR followup, resolution of symptoms of IH paralleled the resolution of the MR findings, including the VDS, which reverted to "absent" in all of the subjects (Fig 3).

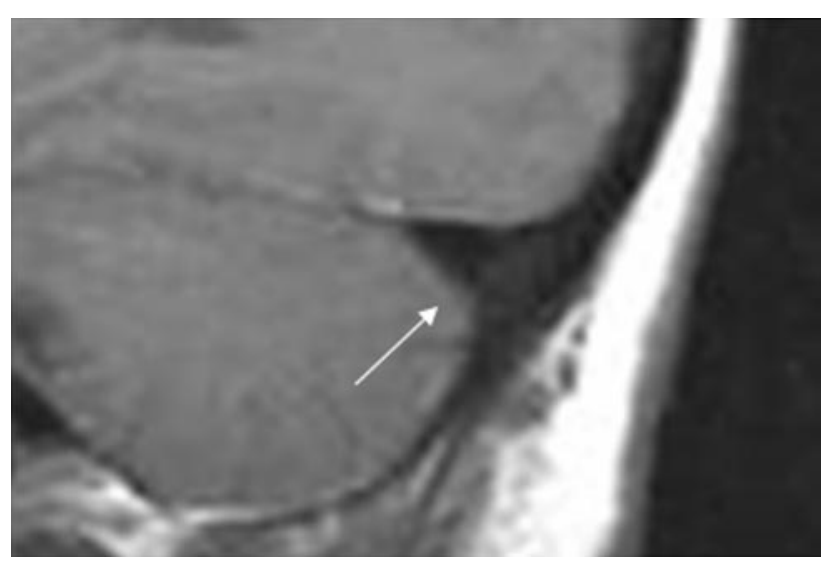

Fig 4. False-negative case. Sagittal T1-weighted image through the approximated middle third of the dominant transverse sinus magnified and cropped of IHP 10. This was interpreted as absent VDS by 2 of the 3 readers and was, therefore, registered as a false-negative result. Note the lower margin of the transverse sinus does appear straight (arrow)

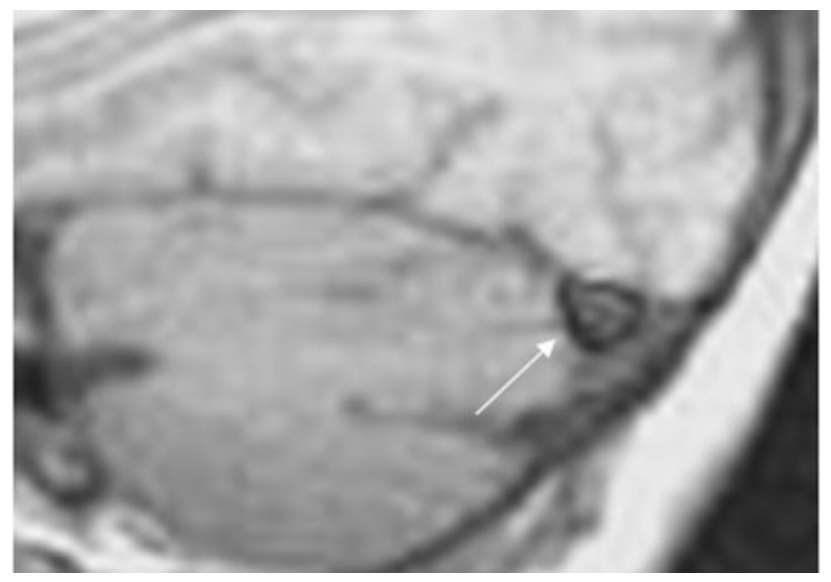

Fig 5. False-positive case. Sagittal T1-weighted image through the approximated middle third of the dominant transverse sinus magnified and cropped of CP 1. This was interpreted as a VDS by all 3 of the readers and, therefore, registered as a false-positive result. Note that the lower margin of the transverse sinus does appear slightly convex (arrow).

\section{Performance of the VDS as a Test}

This sign proved to be a highly accurate test for $\mathrm{IH}$ with a high rate of agreement among the readers (multirater $\kappa=0.82$ ). The overall sensitivity of the sign was $93.3 \%$. The 3 readers correctly identified the IHPs with a VDS in 14 of 15 subjects (unanimously in 12 subjects and by most [ 2 of 3 ] in 2 subjects). In only 1 subject (IHP 10) did the VDS fail to identify a patient with $\mathrm{IH}$, because 2 of the 3 readers tabulated it negative for the sign (Fig 4). Specificity of the sign was $93.3 \%$. The 3 readers correctly identified the CPs with a negative VDS in 14 of 15 subjects (unanimously in 13 subjects and by most in 1 subject). In 1 subject (CP 1), all of the readers incorrectly designated a CP as a patient with IH with a VDS (Fig 5). The 41 remaining (nonage-matched) CPs were reviewed in a nonrandomized fashion by the principal author alone. In all of the subjects the VDS was negative.

\section{Discussion}

The CSF, arterial and venous pressure-volume dynamic, and its effect on the intracranial contents are explained by what has become known as the Monro-Kellie hypothesis. This concept, 
which takes its origins from Monro's ${ }^{16}$ original publication, ${ }^{2,13}$ holds that, in the intact skull, the sum of the volumes of brain, blood, CSF, meninges, and ECF must remain constant. Because the brain itself is not significantly compressible or expandable, a loss of CSF volume must be compensated by an increase in extracellular fluid (ECF) and/or blood volume. These elements also have limitations in accommodating the needed volume. The blood-brain barrier restricts ECF expansion internal to the arachnodural junction; however, significant transudate can accumulate external to this layer of the meninges in patients with $\mathrm{IH}$ and is seen on postgadolinium enhanced T1-weighted imaging as pachymeningeal thickening and enhancement. This accumulation of fluid occurs in the loose connective tissue of the so-called "subdural space." The intracranial venous compartment, in particular, the dural sinuses, can substantially enlarge to compensate for lost CSF volume (resulting in the VDS described here). The MR findings associated with IH (Fig 2) can be predicted by the Monro-Kellie hypothesis and can be categorized as either compensatory or passive. Venous engorgement with enlargement of the dural venous sinuses, enlargement of the pituitary gland, thickening and subsequent enhancement of the loose connective tissue of the subdural space, and transudative fluid collections within this space, as well as frank subdural hematomas are all phenomena that "compensate" for the loss of intracranial volume. The passive findings, which include "slitlike" lateral ventricles and effacement of sulci, as well as the downward transtentorial herniation of the brain with the "sagging brain" appearance, result from the CSF hypovolemia and are further exacerbated by the supratentorial compensatory phenomena. The recognition and interpretation of these MR findings remain qualitative and somewhat subjective. In florid cases of $\mathrm{IH}$, all or most of these findings would probably be present. However, the clinical and MR presentation of this disease is commonly variable $e^{1,3,5,17-23}$ and can occasionally provide a diagnostic challenge; thus, a quantitative reliable sign of venous engorgement may prove useful. We initially noted this VDS at gadolinium-enhanced MR venography in patients with IH and subsequently found that the presence or absence of this sign was easily determined on standard nonenhanced sagittal T1-weighted images.

As a compensatory phenomenon, venous dilation probably occurs early in the course of IH accounting for the high prevalence of this sign in these patients. The concept that the signs of IH appear in a sequential, escalating fashion dependent on the duration and rate of CSF leak has been proposed previously. ${ }^{7,24}$ In a corollary fashion, venous distension is probably the first finding of $\mathrm{IH}$ to disappear on cessation of the CSF leak (after EBP or spontaneous closure), whereas other findings of $\mathrm{IH}$, such as dural enhancement and subdural fluid collections, would require more time to resolve. This concept may explain the single "false-negative" occurrence (Fig 4) in this study (IHP 10). This IHP did have spontaneous resolution of symptoms and may have been imaged shortly after the CSF stopped leaking. This postulate that the MR findings of $\mathrm{IH}$ will ensue and subsequently recede in a predictable sequence may be substantiated in the future. The single falsepositive case in this study is shown in Fig 5. The explanation for this occurrence is uncertain. It may simply relate to normal variation of the contour of the normal transverse sinus and suggests a possible limitation of VDS. The convex contour of the undersur- face of the transverse sinus, however, was not encountered in any of the remaining 41 nonage-matched control subjects.

In summary, evaluation of headache is a common indication for MR of the brain worldwide. IH is increasingly being recognized as a treatable cause of headache that, when left undiagnosed, can lead to prolonged disability. Furthermore, misdiagnosis can lead to unnecessary invasive interventions. IH may not be readily apparent clinically and, thus, not suspected at the time of MR referral. As shown in the present study, the VDS is a reliable sign for the presence of IH that can be applied to the most common and rudimentary of sequences (nonenhanced sagittal T1-weighted image) providing insight into the status of intracranial pressure simply with a glance at the contour of the transverse sinus. In our experience and as shown in the present trial, the VDS appears to be an accurate and potentially helpful imaging sign for the diagnosis of $\mathrm{IH}$.

\section{References}

1. Mokri B. Headaches caused by decreased intracranial pressure: diagnosis and management. Curr Opin Neurol 2003;16:319-26

2. Schievink WI. Spontaneous spinal cerebrospinal fluid leaks and intracranial hypotension. JAMA 2006;295:2286-96

3. Dillon WP, Fishman RA. Some lessons learned about the diagnosis and treatment of spontaneous intracranial hypotension. AJNR Am J Neuroradiol 1998;19:1001-02

4. Mokri B. Syndrome of cerebral spinal fluid hypovolemia: clinical and imaging features and outcome. Neurology 2001;56:1607-08

5. Chung SJ, Kim JS, Lee MC. Syndrome of cerebral spinal fluid hypovolemia: clinical and imaging features and outcome. Neurology 2000;55:1321-27

6. Ferrante E, Savino A, Sances G, et al. Spontaneous intracranial hypotension syndrome: report of twelve cases. Headache 2004;44:615-22

7. Paldino M, Mogilner AY, Tenner MS. Intracranial hypotension syndrome: a comprehensive review. Neurosurg Focus 2003; 15:ECP2

8. Fishman RA, Dillon WP. Dural enhancement and cerebral displacement secondary to intracranial hypotension. Neurology 1993;43:609-11

9. Bakshi R, Mechtler LL, Kamran S, et al. MRI findings in lumbar puncture headache syndrome: abnormal dural-meningeal and dural venous sinus enhancement. Clin Imaging 1999;23:73-76

10. Francia A, Parisi $P$, Vitale AM, et al. Life-threatening intracranial hypotension after diagnostic lumbar puncture. Neurol Sci 2001;22:385-89

11. Bruera OC, Bonamico L, Giglio JA, et al. Intracranial hypotension: the nonspecific nature of MRI findings. Headache 2000;40:848-52

12. Kuhn J, Hofmann B, Knitelius HO, et al. Bilateral subdural haematomata and lumbar pseudomeningocele due to a chronic leakage of liquor cerebrospinalis after a lumbar discectomy with the application of ADCON-L gel. J Neurol Neurosurg Psychiatry 2005;76:1031-33

13. Mokri B. The Monro-Kellie hypothesis: applications in CSF volume depletion. Neurology 2001;56:1746-48

14. Baryshnik DB, Farb RI. Changes in the appearance of venous sinuses after treatment of disordered intracranial pressure. Neurology 2004;62:1445-46

15. The International Classification of Headache Disorders: 2nd edition. Cepha lalgia 2004;24(suppl 1):9-160

16. Monro A. Observations on Structure and Functions of the Nervous System. Edinburgh, UK: Creech and Johnson; 1783

17. Mokri B, Atkinson JL, Piepgras DG. Absent headache despite CSF volume depletion (intracranial hypotension). Neurology 2000;55:1722-24

18. Ferrante E, Savino A. Nonpostural headache by spontaneous intracranial hypotension. Headache 2003;43:127-29

19. Ferrante E, Savino A. Thunderclap headache caused by spontaneous intracranial hypotension. Neurol Sci 2005;26(suppl 2):s155-s157

20. Mokri B, Atkinson JL, Dodick DW, et al. Absent pachymeningeal gadolinium enhancement on cranial MRI despite symptomatic CSF leak. Neurology 1999;53:402-04

21. Kashmere JL, Jacka MJ, Emery D, et al. Reversible coma: a rare presentation of spontaneous intracranial hypotension. Can J Neurol Sci 2004;31:565-68

22. Schievink WI. Misdiagnosis of spontaneous intracranial hypotension. Arch Neurol 2003;60:1713-18

23. Schievink WI, Tourje J. Intracranial hypotension without meningeal enhancement on magnetic resonance imaging. Case report. J Neurosurg 2000;92:475-77

24. Brightbill TC, Goodwin RS, Ford RG. Magnetic resonance imaging of intracranial hypotension syndrome with pathophysiological correlation. Headache 2000;40:292-99 